\title{
Anabases
}

ANABASES Traditions et réceptions de l'Antiquité

$10 \mid 2009$

Varia

\section{La chaîne du savoir. Filiations et parcours intellectuels dans les Sciences de l'Antiquité}

\section{(2) OpenEdition \\ Journals}

Édition électronique

URL : http://journals.openedition.org/anabases/550

DOI : 10.4000/anabases.550

ISSN : 2256-9421

Éditeur

E.R.A.S.M.E.

\section{Édition imprimée}

Date de publication : 1 octobre 2009

Pagination : 27-28

ISSN : $1774-4296$

Référence électronique

"La chaîne du savoir. Filiations et parcours intellectuels dans les Sciences de l'Antiquité ", Anabases

[En ligne], 10 | 2009, mis en ligne le 01 octobre 2012, consulté le 20 octobre 2019. URL : http:// journals.openedition.org/anabases/550 ; DOI : 10.4000/anabases.550 
Anabases 10 (2009), p. 27-28.

\section{La chaîne du savoir Filiations et parcours intellectuels dans les Sciences de l'Antiquité}

«DE Quelque mot Profond tout homme est le disciple», écrivait Victor Hugo; Anabases est assurément fille du voyage et de la «joie spacieuse" que provoque la dilatation du temps et de l'espace, récemment étudiée par Jean-Louis Chrétien. On peut aimer ou détester l'Antiquité: on doit lui reconnaître le mérite d'avoir fécondé la culture moderne et de constituer un objet constamment stimulant et réactif. C'est en ce sens qu'elle nous intéresse au plus haut point. Née en 2004, d'un projet scientifique et culturel ambitieux, la revue Anabases. Traditions et réceptions de l'Antiquité atteint, en 2009, sa dixième livraison. C'est l'occasion de proposer à nos lecteurs un dossier centré sur la thématique des liens intellectuels que la science suscite au fil des générations, des hypothèses et des affinités, à la faveur de la circulation et de la réception des savoirs, et selon des héritages et des relectures.

Fidèle à son orientation transdisciplinaire et interculturelle, Anabases se fait en cela l'écho des objets et des thèmes de recherches en cours au sein de l'équipe ERASME dont elle émane: correspondances scientifiques, biographies intellectuelles, analyses historiographiques, reconstitution des réseaux, histoire des champs et pratiques disciplinaires, étude des comparatismes et des transferts culturels.

Rassemblant l'ensemble de ces pistes de recherche, la question des filiations scientifiques est au centre de l'actualité éditoriale, avec le bel essai de Françoise Waquet sur Les Enfants de Socrate. Filiation intellectuelle et transmission du savoir, XVII $-X X I^{e}$ siècle, paru cette année ${ }^{1}$. Nous nous proposons d'en explorer les ressorts dans le champ spécifique des Sciences de l'Antiquité d'aujourd'hui, en donnant la parole à quelques savants

1 Françoise WAQUET, Les Enfants de Socrate. Filiation intellectuelle et transmission du savoir, $X V I I^{e}-X X I^{e}$ siècle, Paris, Albin Michel, 2009. 
qui ont bien voulu répondre à notre appel (tous n'ont pas souhaité répondre à notre proposition et nous en ont, souvent, expliqué loyalement les raisons. Nous les comprenons, tout en regrettant de ne pouvoir présenter à nos lecteurs des contributions dont nous attentions beaucoup) et se prêter aux exigences - aux joies, nous l'espérons - de l'auto-analyse, à partir des courants de la recherche qu'ils illustrent dans leurs travaux, par leurs parcours personnels et intellectuels, au travers des "généalogies scientifiques", ainsi que des traditions interprétatives qu'ils ont choisies ou accueillies.

Nous les avons invités à nous proposer un essai - une anabase - sur leur filiation intellectuelle, qui soit un moment de réflexivité sur leurs origines et leurs parcours scientifiques, sur les liens qui ont marqué leur itinéraire de recherche, sur les institutions associées à leur carrière, sur la ou les personnalités avec lesquelles ils ont construit un rapport de maître à disciple, ou qui les ont influencés ou marqués dans leur perception de l'Antiquité.

Les membres du Comité de rédaction d'Anabases qui ont joué le jeu de l'egohistoire appartiennent à peu de chose près à la même génération: celle des savants nés pendant ou après la Seconde Guerre mondiale, et arrivés à maturité intellectuelle à partir de la fin des années soixante. Au-delà de leurs origines géographiques diverses, ces spécialistes ont en commun la passion de l'Antiquité, transmise par un ou plusieurs maîtres, et qu'ils s'attachent à diffuser à leur tour auprès de jeunes disciples, entretenant la filiation intellectuelle si bien décrite par Françoise Waquet.

Auteur d'une thèse intitulée Recherches sur le dieu Pan, Philippe Borgeaud enseigne aujourd'hui à l'Université de Genève où il poursuit ses travaux sur les religions antiques. Spécialiste d'historiographie, Hinnerk Bruhns est directeur de recherche au CNRS et se consacre notamment à l'étude comparée de la France et de l'Allemagne. Andrea Giardina officie à l'Istituto italiano di scienze umane, Florence, où il professe l'histoire romaine; il est par ailleurs président de l'institut italien d'histoire antique. Historien de l'Égypte grecque et romaine, c'est à l'Université de Paris I que Bernard Legras forme quant à lui des générations d'étudiants. Né en 1959, Stéphane Ratti est depuis 2000 professeur de langue et littérature latines à l'Université de Bourgogne.

Pour clore le dossier, Corinne Bonnet nous propose une contribution originale: il s'agit de mettre en perspective le témoignage des savants contemporains, en le comparant aux rapports qui unissaient le savant belge Franz Cumont à son maitre allemand Hermann Diels. Si leur relation fut interrompue par la Première Guerre mondiale qui les plaçait de fait dans deux camps opposés, elle reprit plus fortement que jamais par la suite: au-delà de leur profonde affinité intellectuelle, c'est en effet une véritable affection qui unissait les deux hommes. Ce lien affectif est également au cœur des contributions des cinq spécialistes d'antiquité qui se racontent ici : eux aussi reconnaissent la force des sentiments qui les ont liés - et parfois les lient encore - à certains de leurs maîtres. On peut raisonnablement espérer que, contrairement à Cumont, ils n'auront pas pour leur part à surmonter l'épreuve de la guerre...

L'équipe éditoriale 